\title{
Estilos de Aprendizagem dos Estudantes de Medicina e suas Implicações
}

\author{
The Implications of Medical Students' \\ Learning Styles
}

Dejano T. Sobral'

\section{ALAVRASCHAVE:}

Aprendizagem;

- Estudasites de Medicina;

Educação Médica;

Avaliação.

KEY-WORDS

- Learning;

-Medical, Students;

- Medical Education;

- Evaluation.

Recebido em: 20/07/2004

Aprovado em: 06/12/2004

\section{RESUMO}

O propósito do estudo foi analisar as respostas de alunos de Medicina da Ĺniversidade de Brasilia ao Inventário de Estilo de Aprendizagem (IEA) e suas relaçठes com a adaptaçāo inicial ao curso quanto a efeitos no aprendizado e nas opçes e expectativas dos aprendizes. O IEA foi aplicado no terceiro semestre a 911 alunos de ambos os sexos, no decorrer de 14 anos, juntamente com o Questionário de Valorização do Curso e um levantamento da preferência por carreira futura. Os resultados mostraram forte predominância de conceituação abstrata entre os participantes e associaçảo significante do estilo ao sexo, mas nảo à idade. Em conjunto, alunos com estilo concreto (adaptativo - divergente) revelaram menores índices de desenvolvimento pessoal e fortalecimento cognitivo do que os demais, mas sem difereņas no rendimento acadêmico. Observaram-se, também, associą̧ðes significantes entrc cstilo e preferência por carreira. $O$ estilo ativo foi fator preditivo para o exercicio de monitoria, embora bem menos importante do que o rendimento acadêmico global. Os achados demonstraram relaçoes significativas, embora de impacto limitado, entre o estilo de aprendizagem e aspectos de valorização do aprendizado, indicação de preferência por área de atuaçăo e realização de atividade de monitoria.

\section{ABSTRACT}

The purpose of this study was to assess the patterns of medical students' responses to Kolb's Learning Style Inventory (LSI) and their relationship to students' adaptation to the medical program as revealed by learning outcomes and personal choices. The LSI was administered to 911 second-year students of both genders during a 14-year timeframe, together with the Course Valuing Inventory and a survey of initial career preference. The results showed strong predominance of abstract conceptualization among the participants and significant association of style with gender but not age. Overall, concrete-style students (accommodators + divergers) showed weaker scores of personal development and cognitive enhancement than the others, but no loss in academic achievement. An additional finding was the significant relationship between style and career preference. Active style was a predictive factor for peer tutoring activity, although of much less importance than overall academic achievement. In conclusion, the findings evidenced meaningful, albeit small, relationships of individual learning style with features of self-assessment of learning outcomes, early career preference, and peer tutoring activity. 


\section{INTRODUÇÃO}

A identificação do modo individual de aprendizagem do estudante importa à formação profissional? O significado educacional desta indagação se prende, ao menos, a dois aspectos de interesse: a questão da adequação de métodos de instrução c a orientação na escolha de carreira, de acordo com as características dos aprendizes ${ }^{1}$.

Curry ${ }^{1,2}$ identificou distinçōes teóricas entre três categorias de variação individual - preferência instrucional, estilo de aprendizagem e estilo cognitivo -, que estão associadas a caracteristicas de estabilidade temporal e circunstancial. Essa autora propōe as seguintes definiçōes para cada conceito ou construto:

- Preferência instrucional é a afinidade por diferentes modos de acesso à informação ou de sua transmissão;

- Estilos de aprendizagem são comportamentos espontâneos demonstrados, sem escolha ou atenção conscientes, em variedade ampla de situaçōes (de aprendizagem);

- Estilocognitivo diz respeito a consistências individuais em relação à memória c percepção, e ao pensamento e julgamento.

Segundo Sadler-Smith ${ }^{3}$, estilo de aprendizagem é o modo distintivo e habitual pelo qual o aprendiz adquire conhecimento, habilidades e atitudes. Nesse sentido, o estilo se expressa consistentemente em diferentes domínios de conteúdo e pode ser observado em termos de comportamentos típicos.

Estudos realizados em diversos contextos indicam que a idade e, principalmente, o sexo têm efeito no estilo de aprendizagem ${ }^{4,5.6}$. Em contraponto, o estilo de aprendizagem pode influenciar - independentemente de sexo e idade - o desenvolvimento de várias habilidades de entrevista e o grau de rendimento acadêmico ${ }^{7}$. Diferenças no rendimento cognitivo estão relacionadas com a orientação na aprendizagem, em termos do uso de enfoque profundo ou superficial no estudo5. Há evidências, também, de diferenças no estilo de aprendizagem entre profissionais de várias especialidades médicas, particularmente entre cirurgia, psiquiatria e medicina de família ${ }^{8}$.

Existem diversos instrumentos que visam à identificação do estilo de aprendizagem, entre os quais o Inventário de Estilo de Aprendizagem de Kolb tem maior aplicação e divulgação. Esse instrumento se baseia no modelo teórico da aprendizagem vivencial desenvolvido pelo próprio Kolb9,10. O modelo inclui quatro elementos no ciclo vivencial de aprendizagem: (a) vivência concreta; (b) observação e reflexão; (c) formação de conceitos abstratos; (d) experimentação em novas situaçōes. Esses elementos se disporiam segundo duas dimensōes bipola- res (apreensão-conceituação e ação-reflexão), que abarcam funçōes psicológicas distintas, denominadas "compreensão" e "transformação", respectivamente. A validade das duas dimensōes tem apoio em diferentes estudos, embora com ressalvas em alguns aspectos ${ }^{3,11,12}$. Conforme o pólo ou a orientação predominante em cada dimensão, identificam-se quatro estilos básicos de aprendizagem, com implicaçōes adaptativas diversas.

A pertinência das evidências relatadas na literatura internacional para a Educação Médica no Brasil foi o incentivo ao presente trabalho, que faz parte de uma linha de pesquisa sobre caracteristicas da aprendizagem dos estudantes de Medicina c suas implicaçōes para o progresso na formação médica. A questão principal do estudo foi uma apreciação da relação entre o estilo de aprendizagem e a adaptação inicial dos estudantes ao curso, em termos de efeitos no aprendizado pessoal e nas opçōes e expectativas dos aprendizes. Mediu-se o estilo de aprendizagem conforme a primeira versão do inventário elaborado por Kolb甲. O trabalho atual analisa os seguintes objetivos:

- Determinar a distribuição das categorias de estilo de aprendizagem, segundo o inventário de Kolb, entre estudantes de Medicina agrupados por sexo, idade e época de inserção no estudo;

- Examinar as relações entre as categorias de estilo de aprendizagem e três indicadores pertinentes às perspectivas dos estudantes por ocasião da aplicação do inventário: a preferência inicial por carreira, o rendimento acadêmico e a percepção do valor e do significado do aprendizado no primeiro ano de estudos;

- Verificar o efeito potencial dos estilos de aprendizagem dos estudantes na realização subsequiente de atividades de monitoria em disciplinas das ciências básicas.

\section{METODOLOGIA}

\section{Delineamento}

Trata-se de estudo retrospectivo, constituído de dados de coortes consecutivas com elementos de acompanhamento no prazo de dois anos.

\section{Sujeitos}

A população-alvo do estudo compreendeu estudantes do curso de Medicina da Universidade de Brasília, contatados do segundo semestre de 1989 ao primeiro semestre de 2003. O critério de inclusão foi o registro dos estudantes no terceiro periodo cto fluxograma do curso, por amostragem consecutiva de 28 turmas de ingresso semestral ${ }^{13}$. Do total de 911 alunos 
incluídos por esse critério, 10 ( $80 \%$ masculinos) foram excluídos das análises em razão de evasão ainda no terceiro período do curso ou de não terem completado o inventário principal do estudo. Os participantes são oriundos de diferentes regiões do País, cerca de $15 \%$ por transferência de outras faculdades. A média da idade dos 901 alunos credenciados $(54,7 \%$ masculinos) foi $20,59(\mathrm{dp}=2,67)$ no ano de acesso ao terceiro período do curso. A faixa interquartil de idade foi dois anos.

\section{Contexto}

Ao longo do tempo do estudo, os participantes seguiram o novo currículo - de caráter semi-seriado, matrícula por disciplina semestral e regime de créditos - implantado a partir do segundo semestre de 1988. As matérias obrigatórias do fluxo curricular nos primeiros quatro períodos letivos do curso compreendiam principalmente disciplinas da área biomédica e da saúde coletiva. Nesse esquema curricular, os alunos têm múltiplas opções para seguimento de disciplinas optativas, exercício de monitoria em disciplinas já cursadas e atividade de iniciação científica.

\section{Medidas e procedimentos}

Inventário de Estilo de Aprendizagem (IEA). Neste trabalho, utilizou-se a versão em português do Learning Styles Inventory ${ }^{9}$. Um estudo de revalidação mostrou características psicométricas dessa versão semelhantes àquelas do instrumento original ${ }^{14}$. A versão consta de relatório técnico disponível mediante solicitação ao autor.

$\mathrm{O}$ instrumento consiste em um conjunto de nove fileiras de quatro palavras cada. Cada palavra alinhada na fileira denota um modo diferente de aprender, entre os quatros modos identificados por Kolb ${ }^{9,10}$. Esses modos de aprendizagem são os seguintes:

- Orientação para Vivência Concreta (VC, termo-chave: sentindo) - realça o envolvimento em vivências e o trato de situações humanas imediatas de forma pessoal;

- Orientação para Observação Reflexiva (OR, termo-chave: observando) - realça o entendimento do significado de situações e idéias mediante observação e descrição cuidadosas;

- Orientação para Conceituação Abstrata (CA, termo-chave: pensando) - realça o uso de idéias, conceitos e raciocínio;

- Orientação para Experimentação Ativa (EA, termo-chave: agindo) - realça a mudança ativa de situações e a influenciação de pessoas.
O respondente ordena as quatro palavras em cada fileira na seqüência que melhor descreva sua forma de aprender. Para aferir a ênfase relativa em cada orientação de aprendizagem, quatro escores são calculados pela soma do número de ordenação (posto) dos seis termos mais representativos da respectiva orientação. Em acréscimo, dois escores compostos são determinados: um indica a diferença de ênfase entre abstração e concretude (CA-VC); o outro indica a diferença de ênfase entre ação e reflexão (EA-OR). O espaço bidimensional formado pelos dois escores compostos permite identificar quatro estilos individuais - resultantes da combinação de duas orientações predominantes, uma em cada dimensão -, que foram assim denominados: Acomodador ou Adaptativo, Divergente, Assimilador e Convergente. Neste trabalho, a classificação do estilo de aprendizagem baseou-se no valor - positivo, ou negativo - dos escores compostos, segundo o critério proposto por Wunderlich e Gjerde ${ }^{15}$. Utilizou-se o procedimento de análise discriminante para a classificação de casos cujo escore composto teve valor zero.

Questionário de Valorização do Curso (QVC). O QVC é um instrumento de 36 itens, adaptado do Course Valuing Inventory, de Nehari e Bender ${ }^{16}$, mediante versão em português e modificação da terminologia para refletir a perspectiva da vivência curricular global e não somente de uma disciplina isolada. Esse instrumento tem boa consistência interna (alfa de Cronbach $=0,93$ ). Para efeito das análises, foram calculados escores para o total de itens e para conjuntos distintos de itens extraídos por análise fatorial exploratória, conforme descrito em outro trabalho17.

Inquérito sobre a preferência inicial de carreira. A expectativa pessoal sobre a futura carreira em medicina foi obtida mediante a identificação pelo participante da ordem de preferência (1a, 2a e 3a) por área de atuação médica entre 35 opções agrupadas em oito categorias. Uma análise subseqüente levou à classificação dos participantes em cinco grupos de preferência principal: cirurgia geral e especialidades; clínica médica e especialidades; pediatria e tocoginecologia; medicina geral ou alternativa e psiquiatria; e outras (incluindo oftalmologia, ortopedia, radiologia, patologia e anestesiologia).

Registro de monitoria. A purou-se, para cada participante, a ocorrência de atividade de monitoria por dois anos consecutivos (entre o segundo e o quinto período do curso). A realização de monitoria, nessa fase do curso, tem por objeto principal as disciplinas obrigatórias de anatomia, histologia, embriologia, fisiologia, farmacologia, patologia, imunologia, parasitologia e bioquímica e biofísica. 
TABELA 1

Comparação de escores de medidas do Inventário de Estilo de Aprendizagem (IEA) entre estudantes de Medicina no estudo anterior (1992) e no estudo atual (2004)

\begin{tabular}{l|c|c|c|c}
\hline \multicolumn{1}{c|}{ Medidas do IEA } & $\begin{array}{c}\text { Anterior }(N=473) \\
\text { Média }(d p)\end{array}$ & $\begin{array}{c}\text { Atual }(N=901) \\
\text { Média }(d p)\end{array}$ & Teste t \\
\hline VC: Vivência Concreta & $13,87(2,97)$ & $13,48(3,18)$ & 2,26 & 0,024 \\
\hline OR: Observação Reflexiva & $14,88(3,05)$ & $15,74(3,25)$ & 4,76 & 0,000 \\
\hline CA: Conceituação Abstrata & $18,01(2,88)$ & $19,15(3,15)$ & 15,97 & 0,000 \\
\hline EA: Experimentação Ativa & $17,59(3,60)$ & $14,38(3,42)$ & 5,23 & 0,000 \\
\hline CA-VC: Compreensão & $4,12(5,02)$ & $5,67(5,58)$ & 12,39 & 0,000 \\
\hline EA-OR: Transformação & $2,70(5,63)$ & $-1,35(5,99)$ & 0,000 \\
\hline
\end{tabular}

Rendimento acadêmico. $\mathrm{O}$ índice de rendimento acadêmico dos estudantes foi calculado para o primeiro ano e para o terceiro período do curso, com base nas mençôes e no número de créditos das respectivas disciplinas obrigatórias. O gradiente de rendimento cognitivo foi obtido pela comparação emparelhada desses índices consecutivos.

Procedimentos. O IEA foi aplicado na primeira semana do período letivo, como parte de levantamento sobre as condiç̃es e expectativas dos aprendizes, levando em conta a natureza da experiência inicial no curso. Sua aplicação serviu como exercício educativo, e cada aprendiz teve acesso a um retorno comentado de suas respostas, que realçava os diferentes modos de aprender no ciclo vivencial da aprendizagem.

\section{Análise}

Três procedimentos estatísticos principais foram utilizados: (a) testes t, ou análises de variância, para aferir diferenças entre médias de grupos; (b) testes de qui-quadrado, para comparar características ou descritores entre grupos; (c) análise de regressão logística para verificar a associação entre um fator explanatório e a variável binária de desfecho, quando outros fatores são controlados. Os textos de Norman e Streiner ${ }^{18}$ e de Glantz e Slinker ${ }^{19}$ orientaram os planos e procedimentos de análise. A análise foi realizada no programa SPSS 10.0 .

\section{RESULTADOS}

Análises de variância não mostraram diferenças significantes para quaisquer das seis medidas do IEA entre os participantes agrupados segundo a época de inserção no estudo (do primeiro ao sétimo biênio). Não se observou, também, tendência histórica significativa de declínio ou elevação desses escores no decorrer do tempo de estudo.

Foram observadas, contudo, diferenças muito significantes e expressivas entre o conjunto dos participantes do estudo atual (currículo corrente) e do estudo anterior (currículo integrado) para todas as medidas do IEA, exceto vivência concreta. Em conjunto, os participantes do estudo atual mostram nível bem mais positivo na dimensão de "compreensão" (CA-VC̣) e nível bem mais negativo na dimensão de "transformação" (EA-OR). Os dois alunados mostraram distribuição sexual equivalente (teste de Fischer, $p=0,21$ ). A Tabela 1 revela o perfil de diferenças para as seis medidas do inventário.

As análises não revelaram diferenças significantes para as medidas do IEA entre os participantes agrupados por idade. Ocorreram, porém, diferenças significantes entre os sexos nas medidas de vivência concreta (predomínio feminino) e de conceituação abstrata (predomínio masculino). Essas diferenças se expressam na dimensão $\mathrm{CA}-\mathrm{VC}$ - em que o escore do grupo masculino é significantemente maior - e, por conseqüên-

TABELA 2

Comparação de escores de medidas do Inventário de Estilo de Aprendizagem (IEA) entre estudantes de Medicina agrupados por sexo $(\mathrm{N}=901)$

\begin{tabular}{l|c|c|c|c}
\hline \multicolumn{1}{c|}{ Medidas do IEA } & $\begin{array}{c}\text { Homem }(n=493) \\
\text { Média }(d p)\end{array}$ & $\begin{array}{c}\text { Mulher }(n=408) \\
\text { Média }(d p)\end{array}$ & Testet & $p$ \\
\hline VC: Vivência Concreta & $13,22(3,03)$ & $13,80(3,32)$ & $-2,70$ & 0,007 \\
\hline OR: Observação Reflexiva & $15,78(3,18)$ & $15,68(3,34)$ & 0,46 & 0,648 \\
\hline CA: Conceituação Abstrata & $19,56(3,06)$ & $18,65(3,18)$ & 4,37 & 0,000 \\
\hline EA: Experimentação Ativa & $14,27(3,36)$ & $14,52(3,49)$ & $-1,08$ & 0,279 \\
\hline CA-VC: Compreensão & $6,34(5,28)$ & $4,85(5,82)$ & 4,02 & 0,000 \\
\hline EA-OR: Transformação & $-1,51(5,85)$ & $-1,16(6,61)$ & $-0,87$ & 0,387 \\
\hline
\end{tabular}


TABELA 3

Distribuição dos estudantes de Medicina entre quatro estilos de aprendizagem segundo as medidas do Inventário de Estilo de Aprendizagem $(\mathrm{N}=901)$

\begin{tabular}{l|c|c|c|c}
\hline \multicolumn{1}{c|}{ Medidas do IEA } & Adaptativon $=56$ & Divergenten $=92$ & Assimiladorn $=407$ & Convergenten $=346$ \\
\hline VC: Vivência Concreta & $18,39(2,36)$ & $18,51(2,16)$ & $12,97(2,20)$ & $11,95(2,29)$ \\
\hline OR: Observação Reflexiva & $12,30(2,46)$ & $18,27(2,25)$ & $17,82(2,19)$ & $13,16(2,06)$ \\
\hline CA: Conceituação Abstrata & $13,52(2,83)$ & $14,93(2,16)$ & $20,04(2,23)$ & $20,13(2,38)$ \\
\hline EA: Experimentação Ativa & $17,05(2,87)$ & $11,40(2,37)$ & $12,33(2,24)$ & $17,16(2,36)$ \\
\hline AC-CE: Compreensão & $-4,88(4,05)$ & $-3,58(3,15)$ & $7,07(3,39)$ & $8,18(3,59)$ \\
\hline AE-RO: Transformação & $4,75(4,26)$ & $-6,87(3,77)$ & $-5,49(3,33)$ & $4,00(3,30)$ \\
\hline
\end{tabular}

F testes: $p<0,000$ para todas as medidas (anova, one-way).

cia, na distribuição dos quatro estilos de aprendizagem segundo o sexo dos participantes. Observou-se um predomínio feminino nos dois estilos concretos: Adaptativo e Divergente (qui-quadrado $=13,8 ; \mathrm{df}=1 ; \mathrm{p}=0,003$ ). A Tabela 2 mostra as diferenças nos escores das medidas do IEA para o conjunto de participantes agrupados por sexo.

A Tabela 3 revela a distribuição do conjunto dos participantes entre os quatro estilos de aprendizagem, segundo as medidas do IEA e o critério de discriminação. São grupos razoavelmente homogêneos em que predominam os estilos abstratos (Assimilador + Convergente) na dimensão ApreensãoConceituação e os estilos reflexivos (Assimilador + Divergente) na dimensão Ação-Reflexão.

Uma análise de variância não mostrou diferenças significantes no índice de rendimento acadêmico correspondente ao primeiro ano de estudos entre os participantes agrupados conforme $o$ estilo de aprendizagem $(F=0,84 ; d f=3 ; p=0,47)$.

Por outro lado, a Tabela 4 mostra semelhanças e diferenças entre os participantes, agrupados em dois grupos de estilos (abstratos e concretos), nas suas percepções do primeiro ano de estudos mediante as medidas do Questionário de Va- lorização do Curso (QVC). Diferenças significantes, mas discretas, foram observadas nas medidas de desenvolvimento pessoal e de fortalecimento cognitivo do QVC, que favoreceram, em ambos os casos, os estilos abstratos.

Observou-se uma associação significante entre estilo de aprendizagem e preferência inicial por carreira. Duas categorias de preferência - cirurgia e psiquiatria/medicina geral têm posições extremas nas medidas do IEA, especialmente nos escores de observação reflexiva (mais forte na preferência por psiquiatria/medicina geral) e de experimentação ativa (mais forte na preferência por cirurgia). A Tabela 5 mostra a distribuição dos estilos de aprendizagem entre os participantes agrupados em cinco categorias de preferência inicial por carreira, conforme descrito na Metodologia.

O acompanhamento dos participantes revelou tendências distintas no exercício de monitoria nas disciplinas das ciências básicas. Estudantes de estilos Convergente e Divergente mostraram posições opostas na distribuição percentual de atividade de monitoria, com freqüência maior associada ao estilo Convergente. A Tabela 6 revela a distribuição dos estilos de aprendizagem entre os participantes agrupados segundo a

TABELA 4

Diferenças entre as médias das medidas do inventário de valorização do curso (IVC) ao término do primeiro ano de estudos entre estudantes de Medicina reunidos segundo grupos de estilo de aprendizagem $(\mathrm{N}=901)$

\begin{tabular}{|c|c|c|c|c|}
\hline \multirow[b]{2}{*}{$\begin{array}{c}\text { Valorização do Curso } \\
\text { Medidas do IVC }\end{array}$} & \multicolumn{2}{|c|}{ Grupos de estilo de aprendizagem } & \multirow[b]{2}{*}{ Teste t } & \multirow[b]{2}{*}{$p$} \\
\hline & $\begin{array}{c}\text { Abstratos } \\
(n=753) \\
\text { Média }(d p)\end{array}$ & $\begin{array}{c}\text { Concretos } \\
(n=148) \\
\text { Média }(d p)\end{array}$ & & \\
\hline Valimento da vivência & $24,80(2,75)$ & $24,31(3,26)$ & 1,71 & 0,089 \\
\hline Receptividade emocional & $21,32(3,31)$ & $21,48(3,87)$ & 0,51 & 0,645 \\
\hline Desenvolvimento pessoal & $26,79(3,45)$ & $25,99(3,95)$ & 2,51 & 0,012 \\
\hline Fortalecimento cognitivo & $23,08(2,69)$ & $22,57(3,03)$ & 2,04 & 0,041 \\
\hline Disposição de trabalho & $22,69(3,19)$ & $22,13(3,46)$ & 1,94 & 0,052 \\
\hline Pontuação total & $118,69(12,62)$ & $116,49(14,60)$ & 1,71 & 0,088 \\
\hline
\end{tabular}

Estilos abstratos: Assimilador + Convergente. Estilos concretos: Adaptativo + Divergente. 
TABELA 5

Distribuição de estudantes de Medicina segundo categorias de preferência inicial de carreira e grupos de estilo de aprendizagem ( $\mathrm{N}=901$ )

\begin{tabular}{|c|c|c|c|c|}
\hline \multirow{2}{*}{ Preferência principal } & \multicolumn{4}{|c|}{ Grupos de estilo de aprendizagem } \\
\hline & Adaptativo & Divergente & Assimilador & Convergente \\
\hline A. Cirurgia & 17 & 28 & 142 & 148 \\
\hline B. Clínica médica & 20 & 28 & 116 & 102 \\
\hline C. Pediatria/Tocoginecologia & 9 & 20 & 74 & 45 \\
\hline D. Medicina geral/Psiquiatria & 6 & 9 & 34 & 12 \\
\hline E. Outras & 4 & 7 & 41 & 39 \\
\hline Total $(\mathrm{N}=901)$ & 56 & 92 & 407 & 346 \\
\hline
\end{tabular}

Qui-quadrado $=22,1 ; d f=12 ; p=0,036$.

TABELA 6

Distribuição da freqüência de atividade de monitoria realizada por estudantes de Medicina segundo grupos de estilo de aprendizagem $(\mathrm{N}=885)$

\begin{tabular}{l|c|c|c|c|c}
\hline \multicolumn{2}{c}{ Grupos de estilos de aprendizagem } & \multicolumn{5}{c}{ Freqüência de monitoria (a) } \\
\cline { 2 - 6 } & 0 & 1 & 2 & 3 & 4 \\
\hline Estilos ativosAdaptativo $(\mathrm{n}=55)+$ Divergente $(\mathrm{n}=91)$ & 78 & 107 & 120 & 79 & 9 \\
\hline Estilos reflexivosAssimilador $(\mathrm{n}=401)+$ Convergente $(\mathrm{n}=338)$ & 137 & 144 & 135 & 68 & 8 \\
\hline Total $(\mathrm{N}=885)$ & 215 & 251 & 255 & 147 & 17 \\
\hline
\end{tabular}

Qui-quadrado $=12,5 ; d f=4 ; p=0,014$. (a) Acompanhamento por quatro semestres; 16 casos excluidos por seguimento incompleto.

freqüência de exercício de monitoria no prazo de dois anos (do segundo ao quinto período do curso). Observou-se uma associação significante entre estilo ativo de aprendizagem e a freqüência de monitoria no período de acompanhamento.

Análises de regressão logística mostraram que, entre as diferentes medidas e categorias examinadas neste estudo, cinco foram fatores preditivos independentes da realização de monitoria no período de seguimento: índice de rendimento acadêmico no primeiro ano, medida de experimentação ativa do IEA, ordem de preferência inicial por carreira em cirurgia, sexo feminino e medida de fortalecimento cognitivo do QVC, em ordem decrescente de significância. $\mathrm{O}$ modelo de regressão, incluindo os cinco fatores, classificou corretamente a ocorrência, ou não, de monitoria em $79,1 \%$ dos casos. A Tabela 7 resume os dados da análise.

\section{DISCUSSÃO}

Os dados obtidos confirmam que o IEA - na dimensão de "compreensão" - é sensível ao sexo, ou mais propriamente aos valores, atitudes e condutas mais comumente associados

TABELA 7

Análise de regressão logística de fatores de predição da realização de atividade de monitoria em disciplinas das ciências básicas (N = 885)

\begin{tabular}{l|c|c|c|c|c}
\hline \multicolumn{1}{c|}{ Variáveis preditivas } & $\begin{array}{c}\text { Coeficiente } \\
\text { (beta) }\end{array}$ & $\begin{array}{l}\text { Teste } \\
\text { Wald }\end{array}$ & Valor $p$ & $\begin{array}{c}\text { Odds } \\
\text { ratio }\end{array}$ & IC (95\%) \\
\hline Rendimento acadêmico & 1,71 & 86,0 & 0,000 & 5,58 & $3,88-8,02$ \\
\hline Experimentação ativa & 0,09 & 12,7 & 0,000 & 1,09 & $1,04-1,15$ \\
\hline Preferência por cirurgia & 0,23 & 10,7 & 0,001 & 1,26 & $1,10-1,45$ \\
\hline Sexo feminino & 0,50 & 7,1 & 0,007 & 1,66 & $1,14-2,40$ \\
\hline Fortalecimento cognitivo & 0,07 & 5,8 & 0,016 & 1,08 & $1,01-1,15$ \\
\hline (constante) & $-8,67$ & 66,7 & 0,000 & - & - \\
\hline
\end{tabular}

Variável de desfecho: realização, ou não de monitoria no período de quatro semestres.

Wald $=$ teste qui-quadrado $(d f=1)$.

Odds ratio $(O R)=$ razão de probabilidade.

IC = intervalo de confiança de $O R$. 
ao sexo por fatores neurofisiológicos, sociais e culturais. Por outro lado, o fator idade mostrou-se irrelevante, ao menos na estreita faixa etária dos participantes.

Um achado instigante foi a variação significativa nos escores das medidas do IEA na comparação entre o alunado do currículo anterior e o alunado do currículo atual, discrepância realçada pela relativa estabilidade dos escores no decorrer do tempo do estudo atual. Os participantes do estudo atual mostraram uma configuração definidamente mais abstrata e reflexiva nas dimensões do IEA. As diferenças observadas na configuração do estilo de aprendizagem têm possibilidades de explicação distintas, mas não excludentes.

Uma primeira hipótese sugere que a mudança na configuração do estilo de aprendizagem antecedeu o ingresso do aluno no curso. Essa mudança decorreria de variação na composição da população de origem, alteração no filtro do vestibular (grau de exigência, tipo de redação, etc.) ou na atratividade diferencial gerada pela nova imagem do curso (novo currículo, cenário de treinamento, etc.).

A hipótese alternativa sugere que a mudança na configuração do estilo foi gerada a posteriori pela própria renovação curricular. A nova vivência curricular estaria induzindo predominantemente a observação reflexiva e a conceituação abstrata no ciclo de aprendizagem. Na literatura, existem indícios de adaptação comportamental do estilo de aprendizagem à conformação do campo de estudos ao longo da preparação acadêmica ${ }^{20}$.

A segunda hipótese não é favorecida pela constatação de diferenças fracas entre os diversos grupos de estilo de aprendizagem quanto às percepções do valor e do significado do aprendizado no primeiro ano de estudos. Vale dizer, a propósito, que os estudantes cursaram, no primeiro ano, uma gama de disciplinas que não compartilham por certo as mesmas perspectivas epistemológicas e metodológicas. Presumivelmente, a variedade de modos de instrução e opções de aprendizagem do contexto educativo permite acomodar as preferências de instrução associadas aos estilos de aprendizagem ${ }^{3}$.

Contudo, as discretas diferenças observadas nas medidas do QVC entre os grupos de estilo de aprendizagem, particularmente quanto à percepção do desenvolvimento pessoal, interessam por dois motivos: primeiro, são mais nítidas do que as diferenças observadas nos índices de rendimento acadêmico; segundo, independem do rendimento acadêmico, da preferência por carreira e do sexo (dados de análise de regressão, não relatados). Seria de interesse verificar se as discrepâncias nas percepções do significado pessoal do aprendizado entre os estilos concretos e abstratos persistem na fase clínica da formação.
A associação entre estilos de aprendizagem e categorias de preferência inicial por carreira é consistente com o modelo de busca de compatibilidade pessoal na escolha da opção profissional $^{21}$. Segundo Woodward 22 , a imagem inicial da preferência por área de atuação profissional deriva de fatores de gosto, vivência ou interesse econômico, quiçá nessa ordem. $\mathrm{O}$ estilo de aprendizagem, por sua relação com traços da personalidade, possivelmente constitui um dos atributos pessoais de compatibilidade - tais quais sexo - subjacentes ao construto de gosto na influenciação da preferência inicial.

Os dados do exercício de monitoria - que implica escolha pelo aprendiz, ainda que condicionada pelo rendimento acadêmico - sugerem um efeito definido, embora restrito, do estilo de aprendizagem na propensão para realizar a atividade, independentemente de outros fatores contribuintes. O significado potencial dessa associação decorre do impacto positivo do exercício cumulativo de monitoria no desenvolvimento acadêmico e no processo de decisão de escolha do campo de treinamento 23

Os achados deste estudo, em conjunto, sugerem que aspectos da orientação de carreira e de opções de atividade do estudante na fase inicial do curso são influenciados por seu estilo de aprendizagem, interagindo com o sexo, ou não. $\mathrm{O}$ impacto das influências não parece condicionar o aproveitamento das oportunidades de aprendizagem nem a qualidade dos desfechos de aprendizado, exceto em casos extremos de restrição na flexibilidade de adaptação.

O IEA tem uso pedagógico - especialmente em combinação com instrumentos afins ${ }^{24}$ - na medida em que encoraja a reflexão do estudante sobre seu funcionamento cognitivo em função de vivências primárias ou secundárias de aprendizagem ao longo da formação. Os dados compostos de cada turma podem estimular os docentes a oferecer oportunidades alternativas de aprendizagem ${ }^{25}$. O uso do IEA na aferição do estilo de aprendizagem tem a vantagem da rapidez de aplicação, da ordem de poucos minutos. Os alunos de Medicina têm demonstrado continuado interesse na aplicação do instrumento, conforme revela o índice de resposta acima de $99 \%$ no decorrer deste estudo.

Em conclusão, este estudo focalizou dois elementos de análise: a distribuição de estilos de aprendizagem em 28 coortes consecutivos de estudantes de Medicina; e as interações dos estilos com atributos pessoais, desfechos de aprendizado, expectativas e atividades dos participantes. Os resultados apontam relações significativas, mas de impacto limitado, do estilo individual de aprendizagem com sexo, aspectos de valorização do aprendizado, indicação de preferência por área de atuação e realização de atividade de monitoria. 


\section{AGRADECIMENTOS}

$\mathrm{O}$ autor agradece a participação ativa e o interesse real dos aprendizes envolvidos.

\section{REFERÊNCIAS}

1. Curry L. Individual differences in cognitive style, learning style and instructional preference in medical education. In: Norman GR, van der Vleuten CPM, Newble DI, ed. International Handbook of Research in Medical Education. Dordrecht: Kluwer, 2002. p. 263-76.

2. Curry L. An organization of learning style theory and constructs. In: Curry L org. Learning Style in Continuing Medical Education. Ottawa: Canadian Medical Association; 1983. p. 115-31.

3. Sadler-Smith E. The relationship between learning style and cognitive style. Pers Indiv Differ 2001: 30: 609-16.

4. Severiens SE, Tendam GTM. Gender differences in learning styles - a narrative review and quantitative meta-analysis. Higher Educ 1994; 27: 487-501.

5. Hilliard RI. How do medical students learn: medical student learning styles and factors that affect these learning styles. Teach Learn Med 1995; 7: 201-10.

6. Brew CR. Kolb's learning style instrument: sensitive to gender. Educ Psychol Meas 2002; 62: 373-90.

7. Davies SM, Rutledge CM, Davies TC. The impact of student learning styles on interviewing skills and academic performance. Teach Learn Med 1997; 9: 131-5.

8. Curry L. Patterns of learning styles across selected medical specialties. Brit J Educ Psychol 1991; 11: 247-77.

9. Kolb, DA. Learning style inventory technical manual. Boston: McBer, 1976.

10. Kolb, DA. Experiential learning; Experience as the source of Learning and Development. Englewood Cliffs, NJ: Prentice-Hall, 1984.

11. Yahya I. Willcoxson and Prosser's factor analysis on Kolb's (1985) LSI data: reflections and re-analyses. Brit J Educ Psychol 1998; 68: 281-6.

12. Loo R. Confirmatory factor analysis of Kolb's learning style inventory (LSI-1985). Brit J Educ Psychol 1999; 69: 213-9.
13. Hulley SB, Cummings SR. Designing clinical research. Baltimore: Williams \& Wilkins, 1988.

14. Sobral, D.T. Inventário de estilo de aprendizagem de Kolb: características e relação com resultados de avaliação no ensino pré-clínico. Psicol: Teoria Pesq 1992; 8:293-303.

15. Wunderlich R, Gjerde CL. Another look at learning style inventory and career choice. J Med Educ 1978; 53: 45-54.

16. Nehari M, Bender H. Meaningfulness of a learning experience: a measure for educational outcomes in higher education. Higher Educ 1978; 7: 1-11.

17. Sobral DT. Medical students' self-appraisal of first-year learning outcomes: use of the course valuing inventory. Med Teacher 2004; 26: 234-8.

18. Norman GR, Streiner DI. Biostatistics: the bare essentials. St. Louis: Mosby, 1994.

19. Glantz AS, Slinker BK. Primer of applied regression analysis and variance. New York: McGraw-Hill, 1990.

20. Nulty DD, Barrett MA. Transitions in students' learning styles. Studies Higher Educ 1996; 21: 333-45.

21. Burack CJ, Irby DM, Carline JD, Ambrozy DM, Ellsbury KE, Stritter FT. A study of medical students' specialty pathways: trying on possible selves. Acad Med 1997; 72: 534-41.

22. Woodward CA. Developing a research agenda on career choice in medicine. Teach Learn Med 1990; 2: 139.

23. Sobral DT. Cross-year peer tutoring experience in a medical school: conditions and outcomes for student tutors. Med Educ 2002; 36: 1064-70.

24. Sobral DT. Enfoques de estudo de alunos de medicina. Rev Bras Educ Méd 1998; 22: 32-7.

25. Curry L. Cognitive and learning styles in medical education. Acad Med 1999; 4: 409-13.

\section{Endereço para correspondência}

Universidade de Brasília - Faculdade de Medicina CP 04569

70919-970 - Brasília - DF

e-mail: dtsobral@unb.br 